\title{
Akademiker-Erythem
}

Eine 20-jährige Studentin stellte sich mit einer seit zwei Monaten bestehenden netzartigen Hyperpigmentierung an der Vorderseite beider Oberschenkel vor. Sie war sonst gesund und hatte lediglich in den vergangenen sechs Monaten wegen einer Akne vulgaris Isotretinoin eingenommen.

E in Trauma oder ungewöhnliche Gewohnheiten wurden nicht angegeben, an Medikamenten nahm die Patientin aktuell nur orale Kontrazeptiva ein. Bei der genaueren Anamnese konnte man jedoch erfahren, dass sie täglich mehrere Stunden lang den auf den Oberschenkeln ruhenden Laptop benutzte. Dieser Umstand sowie der Lokalbefund führten zur Diagnose eines Erythema ab igne. Dabei handelt es sich um eine gutartige Hyperpigmentierung mit retikulärem Muster, die auftritt, wenn die Haut wiederholt mit Hitze in Kontakt kommt. Sie betrifft etwa $3 \%$ der Bevölkerung und tritt in den meisten Fällen beim Gebrauch von Heizkissen und Wärmflaschen auf.
Große Hitze führt zu Schädigungen der Epidermis entlang der Blutgefäße, wodurch es zu netzartig konfigurierten Hämosiderinablagerungen kommt. Die Zeitdauer bis zum Auftreten des Erythema ab igne ist sehr variabel und kann zwischen zwei Wochen und einem Jahr nach Beginn der Hitzeexposition auftreten.

Außer dem Weglassen der Hitzequelle gibt es keine Behandlungsmöglichkeit. Die Hautveränderungen bilden sich aber in den meisten Fällen innerhalb von Wochen bis Monaten spontan zurück. Bei ausgeprägten Hautschäden wurde die topische Anwendung von 5-Fluorouracil empfohlen.

\section{Globuli gegen Warzen, Pilze, Dermatitis?}

\begin{abstract}
Kann Homöopathie Hautkrankheiten heilen? Nein, sagen die Autoren eines systematischen Reviews nach eingehender Analyse der zwölf verwertbaren kontrollierten Studien zum Thema. Weder Dermatitiden noch Warzen noch Pilze oder offene Beine sprachen in relevantem Ausmaß auf diese Therapie an.
\end{abstract}

$\mathrm{D}$ as belgische Team um Thierry Simonart schloss in seiner Studie sogar alternativmedizinische Publikationen ein, sofern sie kontrolliert waren, sowie nicht englischsprachige und nicht publizierte Untersuchungen. Die Homöopathie wirkte jedoch nicht besser als Placebo weder bei atopischer noch bei strahlenbedingter Dermatitis, weder bei urämischem Juckreiz, noch bei Ulzera an den Beinen und schon gar nicht bei Warzen. Im Fall des rezidivierendem Pilzbefall im Vulvovaginalbereich war die Homöopathie einer konventionellen Behandlung mit Itraconazol deutlich unterlegen.

Signifikante Ergebnisse zugunsten der Homöopathie gab es allerdings in drei Studien (rezidivierende Aphthen der Mundschleimhaut, seborrhoische Dermatitis und atopisches Ekzem). Alle drei Arbeiten wiesen jedoch nach Einschätzung der Reviewer gravierende Qualitäts- mängel auf. In der Studie zur aphthösen Stomatitis waren Schmerzintensität und Umfang der Aphthen nach der homöopathischen Behandlung deutlich geringer als in einer Placebogruppe $(\mathrm{p}<0,05)$. Die Studie war jedoch nur einfach verblindet.

Die kontrollierte Studie zur homöopathischen Therapie der seborrhoischen Dermatitis fiel durch die hohe Drop-outRate auf, die das Ergebnis - eine signifikante Verbesserung im Seborrhoea and Severity Index gegenüber Placebo - stark relativierte.

Bei der Studie zum atopischen Ekzem übertraf der Effekt der Homöopathie auf Zeichen und Symptome (bewertet von Ärzten) sogar den einer konventionellen Behandlung $(\mathrm{p}<0,001)$. Die Studie war jedoch weder verblindet noch randomisiert, die Patienten waren in den Praxen von Homöopathen oder niedergelassenen Ärzten rekrutiert worden und hatten da-
Hyperpigmentierung an den Oberschenkeln nach dem Laptop-Einsatz

Kommentar: Der Schlüssel zur Diagnose lag in diesem Fall einmal mehr in der Anamnese. Der tägliche stundenlange Umgang mit dem Laptop war für die Studentin sicher selbstverständlich und nicht der Rede wert. Wäre sie nicht gezielt darauf angesprochen worden, so hätte sie diese Angabe möglicherweise nicht gemacht.

Prof. Dr. Hermann Füeß।

Botten D et al. Academic branding: erythma $a b$ igne and use of laptop computers. Can Med Assoc J 2010: 182: E857

mit bereits selbst ihre Entscheidung zur Form der Behandlung getroffen. Ein validiertes Scoring-System kam nicht zum Einsatz, die Bewertung wurde durch die Patienten und ihre Behandler vorgenommen.

Fazit: Alles in allem also eine Enttäuschung für die Anhänger der Lehre Hahnemanns. Wo vereinzelte signifikante Ergebnisse Hoffnung aufkeimen lassen, wird diese durch die schlechte Studienqualität gleich wieder zunichte gemacht.

Der Erstautor des Reviews gesteht sich zwar die Limitationen seiner eigenen Studie ein, die letztlich keine härteren Daten liefern kann als die zugrundeliegenden Untersuchungen, und zudem von einem Publikationsbias belastet ist. Trotzdem steht für den Belgier fest, dass man aufgrund der vorliegenden Daten „Homöopathie nicht als evidenzbasierte Therapieform in der Dermatologie ansehen kann."

Dr. Elke Oberhofer

Simonart T et al. Homeopathic remedies in dermatology: a systematic review of controlled clinical trials. Br J Dermatol 2011;165(4):897-905 